\title{
Miniature reaction timer with memory
}

\author{
DAVID HOUGHTON* and ROBERT T. WILKINSON \\ Medical Research Council, Applied Psychology Unit (Annexe) \\ Cambridge $C B 22 B W$, England
}

A pocket-portable instrument is described for the self-administration, in the field, of simple reaction time trials with variable warning foreperiod. The device will store 100 reaction times in milliseconds for later readout in the laboratory, in addition to displaying them to the $S$ at the time of the trial.

For a number of reasons, it may be more difficult to record people's performance in the field than in the laboratory. Three may be distinguished: (1) The necessary apparatus requires power supplies available only in the laboratory or in a limited number of places: (2) the apparatus is too bulky to move into the field or to move about with the $\mathrm{S}$ in the field; and (3) the $\mathrm{E}$ cannot always be available to record performance.

For want of a suitable device, opportunities are often lost for recording standard psychological test performance under the most realistic conditions possible. This is particularly true for studies of environmental stress.

Such a device is described here for measuring simple reaction time with variable warning foreperiod. It is illustrated in Fig. 1. The portable reaction timer with memory consists of a metal box, $12 \times 9.5 \times 5.6 \mathrm{~cm}$, weighing $850 \mathrm{~g}$. The RT can be seen by $\mathrm{S}$ on the display (D) at the time of registration and read out subsequently from the memory by $E$, if $E$ is unable to record the RTs on the spot. The rechargeable batteries allow RTs to be stored periodically for at least $24 \mathrm{~h}$ before any information is lost due to the fall in voltage.

\section{OPERATION}

Before handing the unit over to $S$, the batteries are fitted. The $\mathrm{S}$ can then carry out the first set of RTs as and when directed by his program. He switches on and. holding the unit in his right hand. presses the READY button (R) (Fig. 1) with his first finger. After a delay. which may vary randomly from 0.5 to $3.0 \mathrm{sec}$. the display lights up and starts clocking up in milliseconds. The display lighting up constitutes the stimulus to which $\mathrm{S}$ responds as quickly as possible by pressing the STOP button with the thumb. The display stops and indicates the RT in milliseconds, remaining on for about $2 \mathrm{sec}$. $\mathrm{S}$ may then proceed to the next trial or, indeed, may do so before the display extinguishes if desired. After completing the session of RTs, he switches off. For the succeeding sessions. he proceeds in the same way until the memory is filled with 100 RTs.

*Reprints available from D. Houghton, MRC. Applied Psychology Unit (Annexe), 5 Shaftesbury Road. Cambridge CB2 2BW, England.
If $\mathrm{S}$ fails to operate the timer correctly, the device behaves as follows: When $S$ presses the STOP button before the stimulus occurs, he will score $000 \mathrm{msec}$, which will immediately be displayed. If he does not press the STOP button at all, the count will stop at $1,000 \mathrm{msec}$, but the 1 cannot be displayed. In both these events, the memory will record $000 \mathrm{msec}$. When the STOP button is pressed before the READY button, nothing happens. Now, if the $S$ keeps the READY button continuously depressed, the timer will keep counting up to $999 \mathrm{msec}$ until the button is released. When the button is released, it will count up to 999 . then 000 , and stop, and only one RT of 000 will enter the memory. For these reasons. the system cannot be tricked into giving a false RT.

\section{READOUT FROM THE MEMORY}

Instructions for readout from the memory are as follows: Switch on. Turn the DIRECT/STORE switch to STORE. Press the READY button. If 100 RTs have been entered, the first will now be displayed for $1.0 \mathrm{sec}$. followed by the second for $1.5 \mathrm{sec}$. After this, the display will go dark. Press the READY button again, and the second and third RTs will be displayed before the readout again goes dark, and so on. After 100 RTs have been displayed. the reading will be $000 \mathrm{msec}$ whenever the READY button is pushed. If more than 100 RTs are taken, only the last 100 will be held in the memory. On the other hand. if less than 100 are taken, the first places in the memory will be vacant so that on pressing the button there will be no readout until these blanks have been "pumped" out of the memory by pressing the READY button.

\section{APPARATUS \\ Timer}

Three seven-segment light-emitting-diode (LED) numerical displays. Monsanto Type MAN3A, are used for the timer readout. They have the advantage that they can be operated from the same supply as the logic circuitry: also. LED displays are available in much smaller sizes than those using other techniques. A further feature is that LEDs have no heat-up time, so the lighting of the display can be used as the stimulus for $\mathrm{S}$ to react. Possibly liquid crystal displays have the above advantages together with the advantage of low current consumption, but they were not sufficiently developed at the time of this design.

The displays are driven by seven-segment decoder/drivers. Monsanto Type MSD101, which are made to be used in conjunction with MAN3As and together form a relatively cheap combination. The inputs to the MSDI01s are from three decade counters. Type 7490. or from the memory. The most significant 


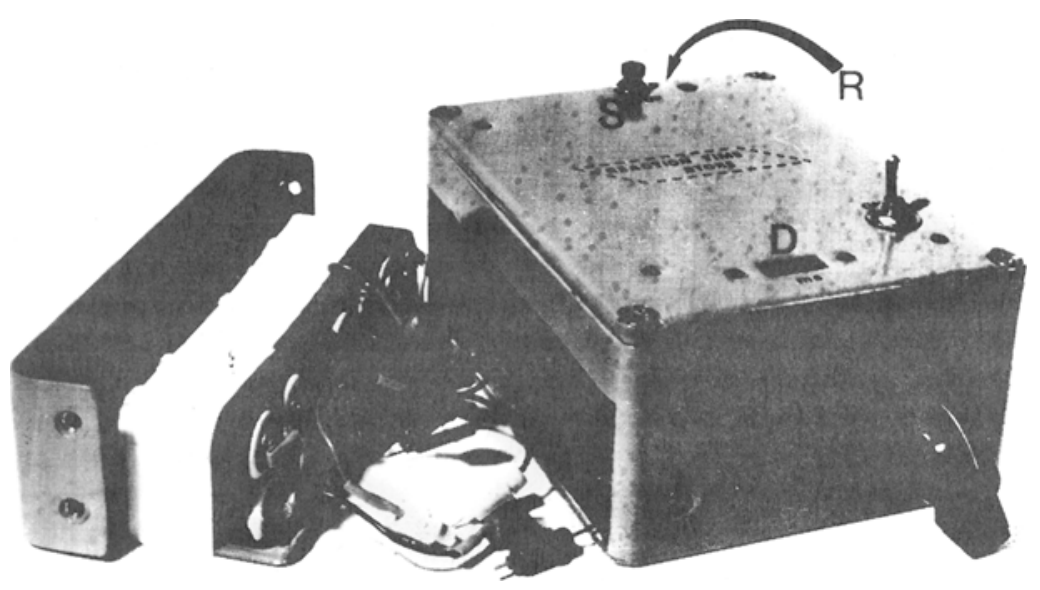

Fig. 1. Reaction time store with battery tray shown removed. decade counter takes its input from the middle decade, and that decade from the least significant decade. This, in turn, has a $1-\mathrm{kHz}$ square-wave input, which is generated by dividing down from a $100-\mathrm{kHz}$ crystal-controlled oscillator. The oscillator circuit uses a C-MOS logic amplifier 1/3 CD4007AE. Two divide-by-10 ICs, Type CD4017AE, divide the oscillator output by a total of 100 . The timer is calibrated by varying the 470-pF capacitor, shown attached to the junction of the $100 \mathrm{k}$ and the crystal, until a frequency meter attached to the oscillator output shows the frequency to be $100 \mathrm{kHz}$.

\section{Control Circuitry}

The control circuitry has the purpose of giving the stimulus, starting and stopping the timer, clocking the digital memory store, and cutting off the supply to the display circuit to limit power consumption.

The function of the READY button is to prime the control circuit by setting the bistable multivibrator $\mathrm{BMV}_{1}$ (Fig. 2). This, in turn, triggers the monostable multivibrator MMV, which resets the decade counters to $000 \mathrm{msec}$ and inhibits the triggering of $\mathrm{BMV}_{2}$ for $0.5 \mathrm{sec}$. After this delay, the first negative-going edge of the free-running astable AMV sets $B M V_{2}$, which allows the timer to start. Simultaneously, $B M V_{2}$ lights the display via one of the diodes of the discrete two-diode AND gate, which switches on the BCY71 and the BFT80, hence supplying power to the three decoder/drivers. Now, AMV has a repetition rate of $2.5 \mathrm{sec}$, so that the delay between the pressing of the READY button and the stimulus lighting up is the sum of $\mathrm{BMV}_{2}$ period, $0.5 \mathrm{sec}$, and the additional time until the next AMV negative pulse. This provides a random delay of 0.5 to $3 \mathrm{sec}$ between READY and stimulus. The
STOP button resets $B M V_{1}$ and, hence, $B M V_{2}$, which inhibits the timer, thus giving the RT, the display being kept alight now by $B M V_{1}$ via the second diode of the discrete component AND gate. The next negative-going edge from AMV sets $\mathrm{BMV}_{3}$, cutting off the supply to the decoder/drivers, thus blacking out the display. It should be noted that before the counter is stopped, $B M V_{3}$ is held in the reset condition by $B M V_{1}$ so that it cannot black out the display before the RT has been displayed. After an RT has been made, periodic operation of the counter by the negative edge of $A M V$ is prevented by the inhibiting action of $B M V_{1}$ on $B M V_{2}$. In the event that the $S$ does not push the STOP button, the 999-to-000 transition of the counter resets $B M V_{1}$, thus stopping the counter.

\section{Memory}

The memory consists of six identical ICs, General Instrument Microelectronics Type SL-7-C2100. These are dual 100-bit static shift registers which take their inputs from the decade counters. There are four binary outputs from each decade counter, which means that two SL-7-C2100s are needed for each decade. Each time $B M V_{1}$ goes into the stop condition (reset condition), the negative edge triggers the memory clock. This acts as a monostable multivibrator and clocks the shift registers, causing the information at the inputs to pass into the registers and all preceding information to be clocked on one place. One hundred RTs may be stored in this manner.

The circuitry as a whole is arranged so that, immediately the batteries are connected, the shift registers are powered with a positive supply of $5 \mathrm{~V}$ and a negative supply slightly greater than $3 \mathrm{~V}$. The supply to the rest of the circuitry is controlled by the ON/OFF 


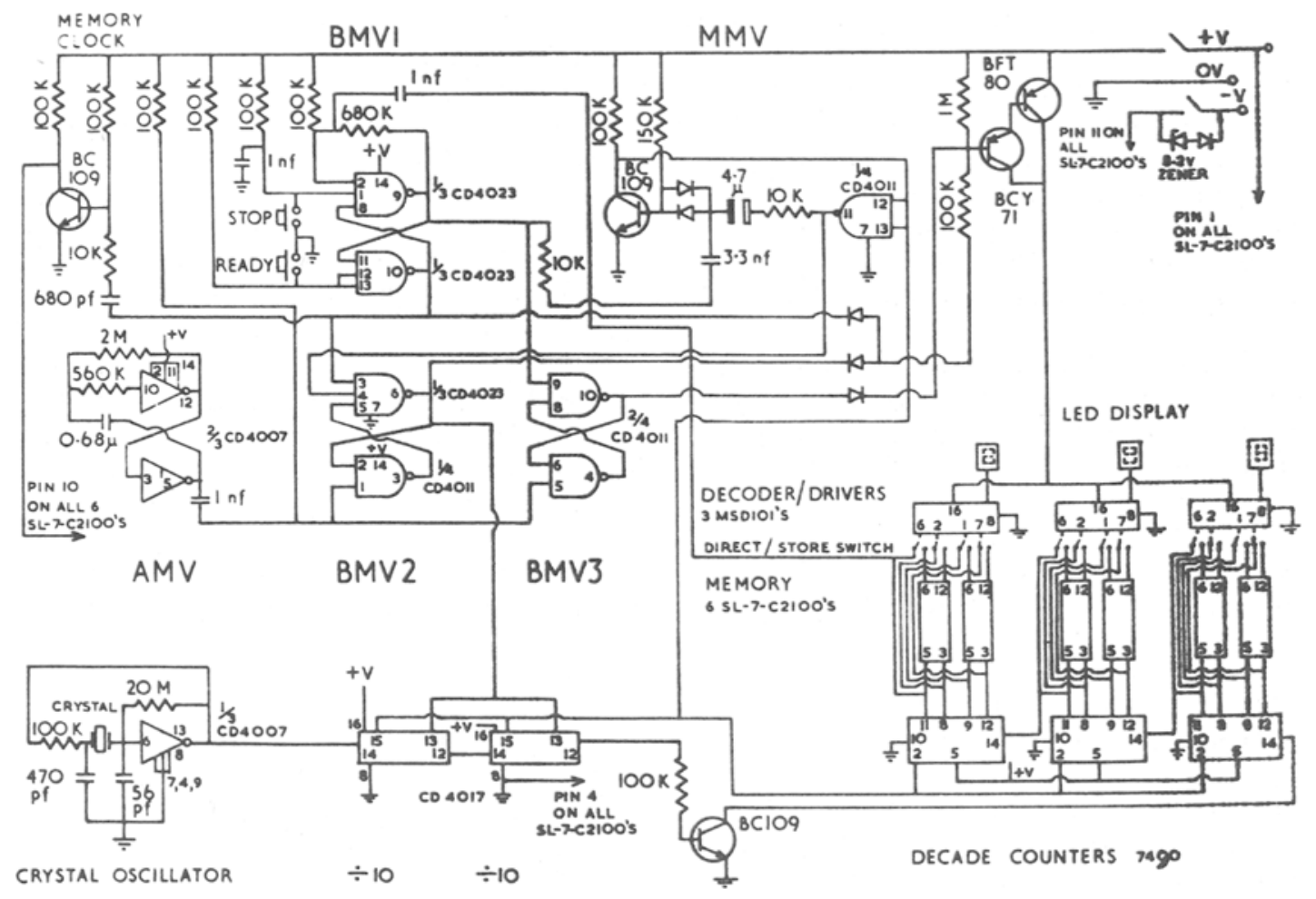

Fig. 2. Circuit diagram of reaction time store.

switch, which also has the function of increasing the shift registers' negative supply from 3 to $12 \mathrm{~V}$ when it is switched $\mathrm{ON}$. This provides a great power saving. because the shift registers will hold their information with a negative $3 \cdot \mathrm{V}$ supply but they need $-12 \mathrm{~V}$ when being clocked and when giving an output.

A miniature 12-pole two-way switch effects the changeover of the display from direct to memory.

\section{Components}

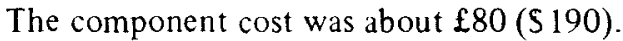

The details of the manufacturers of components are given only as an aid in the event of difficulty in obtaining components. Practically all the components. with the exception of the C-MOS logic. should be obtainable from many sources.

All the resistors are $5 \%$ tolerance. Due to difficulty in obtaining a small 22-M resistor. the 20-M resistor in the crystal oscillator circuit is made up from two 10-M resistors in series. however. a 22-M resistor may be used if available in small size.

The capacitors used in the crystal oscillator are mica $1 \%$ tolerance. Other capacitors are small-size toil types apart from the $4.7 \mu \mathrm{F}$ used in the MMV circuit. which is a dry tantalum electroly tic

Where possible, R.C.A. complementary MOS logic is used, because it consumes less power than TTL or DTL. However. in the case of the decoder/drivers and decade counters, no equivalent C-MOS logic was available at the time of design.
Discrete components are used instead of IC logic, either where there is a need for more current capability

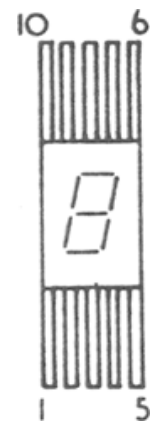

\begin{tabular}{|c|c|c|}
\hline MAN $3 A$ & & MSDIOI \\
\hline Pin No. & to & Pin No. \\
\hline 1 & & 9 \\
\hline 2 & & 10 \\
\hline 3 & & Or line \\
\hline 4 & & 11 \\
\hline 5 & & N.C. \\
\hline 6 & & 12 \\
\hline 7 & & 13 \\
\hline 8 & & Or line \\
\hline 10 & & 15 \\
\hline
\end{tabular}

Fig. 3. Wiring connections between MAN3A and MSD101. 
than that afforded by C.MOS or where only one gate is needed and IC logic would take more space.

The memory components were chosen to compromise between size and cost. For example, a digital tape recorder was rejected on grounds of size. Technically, C-MOS shift registers are preferable to the ones chosen, as they need only one power supply and consume less power, but the cost of a C-MOS 64-bit static shift register is four times that of the dual 100-bit register chosen.

A quartz crystal unit ${ }^{1}$ is used to provide good oscillator stability. There are many sources of such crystals, the specification being a high-Q $100-\mathrm{kHz}$ crystal to be used in series resonance, with a circuit capacity of $50 \mathrm{pF}$.

Texas Instruments supply the BCY71, BFT80, and BCI09 transistors. The diodes are BAX13s, but any general low-power silicon diode will be suitable.

\section{Batteries}

Saft-Nickel-Cadmium ${ }^{2}$ rechargeable cells provide the power. Four Type VR0S cells are used in series for the 5.V supply and 10 in series for the $12-\mathrm{V}$ supply. They last about $24 \mathrm{~h}$ from the time of plugging in. For ease of accessibility, the batteries are taped with plastic insulation tape to a tray which slides into the side of the die-cast box, and is secured with four screws.

\section{Housing}

The unit is housed in a die-cast box measuring $4{ }^{3}+\mathrm{x}$ $33.4 \times 21 / 4$ in., which means that it can comfortably be held in one hand. The READY and STOP buttons are positioned so that they can be operated by the first finger and thumb, respectively, of the hand supporting the box. Just above the battery compartment is the circuit board, which is mounted on spacing pillars from the lid of the box. The lid has a window cut in, which allows viewing of the LED display.

\section{MODIFICATIONS FOR THE FUTURE}

Both RCA and Motorola are expanding their ranges of C-MOS devices and, subject to availability and price reductions, it should be feasible to replace the present memory with dual C-MOS shift registers. The $-12 \cdot \mathrm{V}$ supply will not then be required, thus allowing further reduction in size or, alternatively, an expansion of the memory store.

\section{NOTES}

1. The present one was obtained from the Quartz Crysta Company Ltd., QCC Works, Wellington Crescent, New Malden, Surrey, England.

2. Saft Corporation of America, 50 Rockefeller Plaza, New York, New York 10020.

(Received for publication October 10, 1972; revision accepted November 15,1972 .) 